\title{
Synaptic Transmission Mediated by Single Club Endings on the Goldfish Mauthner Cell. II. Plasticity of Excitatory Postsynaptic Potentials
}

\author{
Jen-Wei Lin and Donald S. Faber \\ Department of Physiology, State University of New York at Buffalo, Buffalo, New York 14214
}

Simultaneous pre- and postsynaptic intracellular recordings were used to analyze the properties of chemically mediated synaptic transmission between single club endings of eighth nerve afferents and the goldfish Mauthner (M-) cell lateral dendrite. The EPSPs exhibited pronounced facilitation when the presynaptic fiber fired high-frequency bursts of 2 or 3 impulses at intervals of 2-4 msec. The amplitudes of the EPSPs evoked by the second and third presynaptic impulses of a burst were, on average, 99 and $108 \%$ larger than that evoked by the first impulse. A cross-correlation analysis showed that the amplitudes of the control and facilitated EPSPs fluctuated independently, indicating that the facilitation was mediated by a presynaptic mechanism. This conclusion was supported by a comparison of the coefficient of variation for the control and facilitated EPSPS, on the basis of a binomial release model. In addition, the value of binomial $n$, the number of presynaptic release units, was not changed during facilitation.

The origin of EPSP fluctuations was analyzed by examining the correlation between the amplitudes of EPSPs and those of the electrotonic coupling potentials associated with them. The absence of correlation between the 2 variables suggested that the fluctuations of EPSPs were not due to a variable presynaptic impulse invasion. The EPSP fluctuations were further analyzed by assuming that the facilitation was associated with an increase in the probability $(p)$ of transmitter release and that the release process followed simple binomial statistics. The binomial variables thus calculated were $n=6-11, p=0.29-0.44$, and $q=31-61 \mu \mathrm{V}$, values comparable to the estimates for other CNS synapses. More importantly, these parameters provided satisfactory fits to the amplitude histograms of the control and facilitated EPSPs. The number of release units, $n$, was smaller than, but in a range similar to, the number of active zones identified in the freeze-fracture study of the club endings (Kohno and Noguchi, 1986). This correlation is consistent with the notion that active zones are the structural correlates of quantal release units.

\footnotetext{
Received Apr. 28, 1987; revised Sept. 21, 1987; accepted Sept. 23, 1987.

We thank Julie Lakatos for graphics, Jan Jordan for typing the manuscript, and Dr. M. V. L. Bennett for critical comments during these studies. This work was supported in part by NIH Grants NS15335 and NS21848.

A portion of this research was submitted by J.-W. L. in partial fulfillment of the requirements for the degree of Doctor of Philosophy at the State University of New York, Buffalo, NY.

Correspondence should be addressed to Dr. Faber, Department of Physiology, Neurobiology Laboratory, State University of New York, 313 Cary Hall, Buffalo, NY 14214.

Copyright (C) 1988 Society for Neuroscience $0270-6474 / 88 / 041313-13 \$ 02.00 / 0$
}

In the preceding paper, it was shown that impulses in a majority of club endings electrotonically coupled to the $M$-cell do not produce a detectable chemically mediated EPSP, although the contacts have the morphological correlates of chemical synapses. In an attempt to activate these "silent" connections, 2 approaches were used. First, the burst-firing paradigm, which could effectively facilitate EPSPs already present, falled to reveal any EPSPs at the silent junctions. Second, intracellular injection of 4-AP (or Cs) into the saccular fibers increased the percentage of the fibers that mediated chemical transmission, from less than 20 to $56 \%$. Furthermore, the potency of the "unmasking" effect produced by 4-AP and Cs was paralleled with their ability to broaden the duration of the presynaptic impulses. The average half-width of coupling potentials due to presynaptic action potentials increased from $242 \mu \mathrm{sec}$ in those afferents studied with $\mathrm{KCl}$ electrodes to $254 \mu \mathrm{sec}$ with $\mathrm{CsCl}$ electrodes and to $320 \mu \mathrm{sec}$ with 4-AP. There was a clear correlation between the input, presynaptic impulse duration, as indicated by the half-width of the coupling potentials, and the output, EPSP amplitude. We conclude that the chemical synapses in the club endings operate in the lower region of their input-output curve, and a small increase in presynaptic impulse duration can increase transmitter output from effectively zero to a detectable level.

The findings presented in the preceding paper (Lin and Faber, 1988) demonstrate that saccular nerve fibers which terminate on the Mauthner (M-) cell lateral dendrite as large myelinated club endings have certain unique features that can be exploited for the analysis of chemical transmission in the CNS. First, these fibers send only one branch, with one terminal, to the M-cell, and since these elements are electrotonically coupled, the coupling potential amplitude is an independent measure of the presynaptic action potential. Thus, it is possible to determine directly whether fluctuations of unitary EPSPs are associated with variations in impulse invasion of the presynaptic terminal, such as might be associated with failure of active spike propagation at axonal branch points or nodes (Luscher et al., 1979, 1983a; Henneman et al., 1984). Second, as is described in more detail in the present paper, these excitatory synapses exhibit a marked facilitation of transmission when the presynaptic fibers are activated repetitively at short intervals. Although short-term plasticities of synaptic transmission such as facilitation, depression, and posttetanic potentiation (PTP) have been demonstrated in various regions of the vertebrate CNS (Curtis and Eccles, 1960; Porter, 1970; Lomo, 1971; Korn et al., 1984), detailed and 
mechanistic investigations of these properties have been provided in only a few instances. Results thus far from such diverse preparations as the crayfish neuromuscular junction, inhibitory synapses on the M-cell, and Ia-motoneuron connections all suggested that the probability, $p$, of release of transmitter (Zucker, 1973; Hirst et al., 1981; Korn et al., 1984) may be the variable modified during these short-term synaptic plasticities. However, although the evidence is quite compelling that changes in branch point failure do not underlie those observations (Jack et al., 1981a; Korn et al., 1981, 1982, 1984), this concept as well has been proposed to account for both PTP and the EPSP fluctuations observed at Ia-motoneuron connections (Luscher et al., $1979,1983 a)$. In the present report, we present evidence that the facilitation of EPSPs mediated by the club endings has a presynaptic origin and, furthermore, neither facilitation nor the fluctuations in synaptic transmission observed with low-frequency stimulation are correlated with changes in the presynaptic spike amplitude.

All club endings that have been studied at the ultrastructural level establish multiple chemical synapses on the lateral dendrite, as identified by discrete active zones and related pre- and postsynaptic specializations (Nakajima, 1974; Nakajima and Kohno, 1978; Kohno and Noguchi, 1986). Yet more than $80 \%$ of the connections studied electrophysiologically appeared to be functionally silent in that presynaptic impulses did not produce detectable EPSPs (Lin and Faber, 1988). Evidence for silent connections in other systems has been generated by indirect means in that experimental manipulations such as spinal cord (Nelson et al., 1979) and dorsal root (Dostrovsky et al., 1976; Millar et al., 1976) transections have unmasked previously undetected connections. The mechanisms and locus of this functional block, which necessarily operates at the level of all the synapses between an afferent and its target cell, have heen explored in the present study. We report that many, but not all, silent connections can be unblocked by presynaptic injections of 4-aminopyridine (4-AP), which produce very slight increases in the duration of the terminal action potential, while the repetitive stimulation paradigm, which produces facilitation at transmitting connections, is ineffective.

Combined morphophysiological analyses of inhibitory synapses on the $\mathrm{M}$-cell have been interpreted as indicating that the amplitudes of postsynaptic potentials produced by single presynaptic impulses are composed of integral multiples of elementary voltage steps (quanta, $q$ ) and that individual terminal boutons are the basic units that produce such steps, each having the same average probability, $p$, of transmitter release (Korn et al., 1981, 1982). In that system, amplitude histograms of unitary inhibitory postsynaptic potentials could be fit by a simple binomial model and $n$, the number of available quanta release units, was equivalent to the number of morphologically identified terminal boutons established by the presynaptic neuron on the M-cell. Ultrastructural evidence that each ending contained only one active zone (Triller and Korn, 1982) led to the conclusion that a single presynaptic grid was the ultrastructural correlate of the quantal unit (see also Zucker, 1973). In 2 other systems, the Ia to motoneuron connection of cat spinal cord (Jack et al., $198 \mathrm{la}$; Redman and Walmsley, 1983) and synapses between cultured spinal neurons (Neale et al., 1983; Nelson et al., 1983), a somewhat different conclusion was reached, namely, that the terminal bouton is a quantal unit, despite the fact that one such ending has multiple active zones. This alternative notion was based on the finding that in those systems the value of binomial $n$ was the same or less than the number of morphologically identified terminals. It requires that a terminal with multiple active zones nevertheless releases transmitter in an allor-none manner or that a single quantum released at any one site saturates the receptors at the corresponding postsynaptic locus and, necessarily, at all adjacent synapses established by the same terminal (Neale et al., 1983; Redman and Walmsley, 1983; Pun et al., 1986). The present investigation of synapses established by single club endings allowed this alternative hypothesis to be addressed directly. Two approaches to quantal analysis of fluctuations in unitary EPSP amplitudes indicate that, in this system, binomial $n$ is appreciably greater than 1; that is, the quantal unit is not the terminal, which in this system also contains multiple active zones.

\section{Materials and Methods}

The techniques used for anesthesia, surgery, and obtaining simultaneous intracellular recordings from single saccular nerve afferents and the lateral dendrite of the M-cell were as described in the preceding paper (Lin and Faber, 1988). In some experiments, the presynaptic electrode contained either $0.1 \mathrm{~m}$ 4-aminopyridine (4-AP; Sigma) in $1.25 \mathrm{M} \mathrm{KCl}$ solution or $2 \mathrm{M} \mathrm{CsCl}$. Electrodes filled with these solutions had final resistances of $30-40 \mathrm{M}$.

Binomial analysis of EPSP amplitudes. Binomial statistics were used in the quantal analysis of the EPSPs evoked by impulses in the saccular fibers. The binomial model for quantal release depends on the following assumptions: (1) the single terminal from a presynaptic cell has $n$ release units; (2) all the release units of one cell have a uniform probability $(p)$ of transmitter release; (3) each time the quantal unit secretes transmitter, it produces a constant postsynaptic response with an amplitude of $q$ microvolts $(\mu \mathrm{V})$, called quantal size; and (4) exocytosis at individual release units is independent and follows a random process (McLachlan, 1978). If variations in $n, p$, and $q$ are not taken into consideration, the basic relationships between the binomial parameters and experimental data are

$$
\begin{aligned}
& \bar{x}-n p q, \\
& v=n p(1-p) q^{2},
\end{aligned}
$$

where $\bar{x}$ and $v$ are the mean amplitude and variance of the EPSPs, respectively.

When transmitter release is facilitated due to an increase in the release probability, from $p_{1}$ to $p_{2}$, the means and variances of the control and facilitated EPSPs can provide a solution for the variables, $n, q, p_{1}$, and $p_{2}$ (see also, Nelson et al., 1983). Specifically, there are 4 simultaneous equations to solve:

$$
\begin{aligned}
& \bar{x}_{1}=n p_{1} q, \\
& v_{1}=n p_{1}\left(1-p_{1}\right) q^{2}, \\
& \bar{x}_{2}=n p_{2} q, \\
& v_{2}=n p_{2}\left(1-p_{2}\right) q^{2},
\end{aligned}
$$

where $\bar{x}_{1}$ and $\bar{x}_{2}$ are the mean amplitudes of the control and facilitated EPSPs, respectively, and $v_{1}$ and $v_{2}$ are the corresponding variances. The resulting expressions for the binomial variables are

$$
\begin{aligned}
n & =\left(\bar{x}_{1}-\bar{x}_{2}\right) /\left(v_{2} / \bar{x}_{2}-v_{1} / \bar{x}_{1}\right), \\
p_{1} & =\left(\bar{x}_{1} / \bar{x}_{2}-v_{1} / v_{2}\right) /\left[\bar{x}_{1} / \bar{x}_{2}-\left(v_{1} / v_{2}\right)\left(\bar{x}_{2} / \bar{x}_{1}\right)\right], \\
p_{2} & =\left(\bar{x}_{1} / \bar{x}_{2}-v_{1} / v_{2}\right) /\left[\left(\bar{x}_{1} / \bar{x}_{2}\right)^{2}-v_{1} / v_{2}\right], \\
q & =\left[\left(v_{2} \bar{x}_{1}\right) / \bar{x}_{2}-\left(v_{1} \bar{x}_{2}\right) / \bar{x}_{1}\right] /\left(\bar{x}_{1}-\bar{x}_{2}\right) .
\end{aligned}
$$

It should be stressed that these solutions only pertain if $n$ and $q$ are unchanged during facilitation and if $p$ is uniform across all release sites in each experimental condition. In contrast, the binomial parameters cannot be extracted by a similar approach if facilitation is due to an increase of $n$, from $n_{1}$ to $n_{2}$, while $p$ and $q$ remain constant. However, we can test for this possibility by taking the ratios of the means and variances of the EPSPs, as indicated below: 


$$
\begin{aligned}
& \bar{x}_{1} / v_{1}=n_{1} p q / n_{1} p(1-p) q^{2}=1 /[(1-p) q] \\
& \bar{x}_{2} / v_{2}=n_{2} p q / n_{2} p(1-p) q^{2}=1 /[(1-p) q]
\end{aligned}
$$

That is, if only $n$ changes during facilitation, the ratio $\bar{x}_{1} / v_{1}=\bar{x}_{2} / v_{2}$.

The coefficient of variation (CV) can also provide clues to the possible origin of the facilitation. Specifically, the CV has the following relationship to the binomial variables:

$$
\begin{aligned}
\mathrm{CV}=\sqrt{(v) / \bar{x}} & =q \sqrt{n p(1 \quad p)} / n p q \\
& =\sqrt{(1-p) / \mathrm{n} p}
\end{aligned}
$$

If facilitation is mediated by a presynaptic mechanism, due to an increase in either $p$ or $n$ or both, the CV of the facilitated responses will become smaller than that of the control responses. In contrast, a postsynaptic facilitatory mechanism will be associated with an increase of $q$, which is not a variable in equation (12), and the $\mathrm{CV}$ should not change during facilitation. These expectations will pertain even if there are randon variations in $n, p$, or $q$ (see below).

All signal variances were corrected for background noise, as described in the companion manuscript (Lin and Faber, 1988). The corrected variance was then used for the binomial analysis. The time window at which the potentials were measured remained constant for both noise and signal such that the "bandwidth" of the measurements was the same. In some experiments, the variance measured with calibration pulses was compared with that measured from baseline. The latter consistently had a mean of zero and the same variance as that obtained from the calibration pulse measurements.

The preceding deviations were based upon the assumptions that the parameters $n, p$, and $q$ remained invariant for a given stimulus condition, and recent studies have indicated that quantal size is quite constant at a number of central synapses (see Korn et al., 1987). Nevertheless, in view of the indirect analyses required here, we considered the effects of allowing variations in each parameter, one at a time. The corrected expressions for the signal variances and for the individual parameters are listed below.

1. Variance in quantal size, $v_{q}=\left(\alpha_{q}\right)^{2} \bar{q}^{2}$ (McLachlan, 1978):

$$
\begin{aligned}
v & =n p(1-p) q^{2}+n p v_{q} \\
n_{q} & =n \\
p_{1, q} & =p_{1}\left[1+\left(\alpha_{q}\right)^{2}\right] \\
p_{2, q} & =p_{2}\left[1+\left(\alpha_{q}\right)^{2}\right] \\
q_{q} & =q\left[1+\left(\alpha_{q}\right)^{2}\right]^{-1}
\end{aligned}
$$

2. Variance due to spatially nonuniform release probability $\left[v_{p}=\left(\alpha_{p}\right)^{2} p^{2}\right]$ :

$$
\begin{aligned}
y & \approx n \bar{p}(1-\bar{p}) q^{2}-v_{p} n \\
n_{p} & \approx n\left[1+\left(\alpha_{p}\right)^{2}\right] \\
p_{1, p} & \approx \bar{p}_{1}\left[1+\left(\alpha_{p}\right)^{2}\right]^{-1} \\
p_{2, p} & \approx p_{2}\left[1+\left(\alpha_{p}\right)^{2}\right]^{-1} \\
q_{p} & \approx q
\end{aligned}
$$

3. Variance due to random temporal fluctuations in the number of quantal release units, $v_{n}=\left(\alpha_{n}\right)^{2} \bar{n}^{2}$ (McLachlan, 1978):

$$
\begin{aligned}
v & =\bar{n} p(1-p) q^{2}+v_{n} p^{2} q^{2} \\
n_{n} & =\bar{n}\left[1+\left(\alpha_{n}\right)^{2} \bar{n}\right]^{-1} \\
p_{1, n} & =p_{1}\left[1+\left(\alpha_{n}\right)^{2} \bar{n}\right] \\
p_{2, n} & =p_{2}\left[1+\left(\alpha_{n}\right)^{2} \bar{n}\right] \\
q_{n} & =q
\end{aligned}
$$

The lettered subscripts refer to the parameter subject to variation, $v_{n}$ and $\alpha_{n}$ are the corresponding variance and CV for $n$, etc., $n, p_{1}, p_{2}$, and $q$ are as defined in equations $7-10$, and $\bar{n}, \bar{p}$, and $\bar{q}$ are their corresponding mean values. It should be recognized that these relationships were derived for the specific circumstances pertaining to the experiments reported here and therefore differ from other theoretical treatments where quantal size could be taken as equal to the amplitude of miniature potentials (Brown et al., 1976; McLachlan, 1978).

\section{Results \\ Facilitation of EPSPS}

The EPSPs evoked by impulses in single club endings exhibited facilitation when the presynaptic fiber fired a high-frequency burst. Figure $1 A$ presents an example of 3 presynaptic impulses evoked by an $8 \mathrm{msec}$ depolarizing current pulse; facilitation of the second and third EPSPs is apparent. Amplitude histograms of the first $\left(\mathrm{EPSP}_{1}\right)$ and second $\left(\mathrm{EPSP}_{2}\right)$ responses are shown in Figure $1 B$. The average amplitude of the second EPSP was more than twice that of the first. To quantify the magnitude of facilitation, the facilitation coefficient $\left(F_{l}\right)$ of the $i$ th EPSP is defined as

$$
F_{i}=\left[\left(\mathrm{EPSP}_{i} / \mathrm{EPSP}_{1}\right)-1\right] 100 \%
$$

where $\operatorname{EPSP}_{i}$ and $\operatorname{EPSP}_{1}$ are the average amplitudes of the $i$ th and first EPSPs, respectively. For interspike intervals of 1.6-4 msec, $F_{2}$ varied over a large range, from 27 to $164 \%$, and averaged $99.0 \pm 40.2 \%(n=14)$. The range of $F_{3}$ was comparable, $12-223 \%$, and with a mean of $107.9 \pm 93.9 \%(n=8)$, which is only slightly larger than that of $F_{2}$. The interspike intervals for $F_{3}$, defined between the first and the third impulses, ranged from 3.5 to $7 \mathrm{msec}$.

The full time course of facilitation was not characterized in these experiments since stable intracellular recordings from a saccular fiber were difficult to maintain for the time required to achieve such an analysis. Nevertheless, facilitation generally (1) was not apparent when the fibers were stimulated at constant frequencies of less than $20 \mathrm{~Hz},(2)$ increased progressively as the interspike interval decreased during the burst, and (3) was maximal at the shortest interval tested, $1.6 \mathrm{msec}$. Finally, the waveform of the facilitated EPSPs appeared to be the same as that of the control ones.

Correlation of control and facilitated EPSPS. To investigate the possible mechanisms underlying this facilitation, we first asked whether the amplitudes of the first and second EPSPs were correlated. A positive correlation would suggest that the effect is mediated by a release-dependent mechanism, such as receptor sensitization. Specifically, if the amplitudes of EPSP were dependent upon the amount of transmitter released by the first impulse, a positive correlation would be expected. The same result might also occur if release was enhanced only at those active zones that had undergone exocytosis after the first impulse. On the other hand, if the enhancement was due to a uniform increase in the release probability at all active sites and if release was indeed a random process, no correlation between EPSP $_{1}$ and EPSP $_{2}$ would be expected. Other mechanisms arc possible, but it can be stated generally that a release-dependent mechanism, which could have a pre- or postsynaptic origin, would result in a positive correlation, whereas a release-independent mechanism, which should be of presynaptic origin, would not.

The cross-correlation analysis was performed in 6 experiments, and a statistically significant correlation was never observed. An example is shown in Figure 2, where the lack of correlation is first demonstrated by the independent fluctuations of the first and second EPSPs in the single traces of $A$. When the amplitude of the second EPSP was plotted against that of the first one (Fig. $2 B$ ), the scatter of the data points suggested the 2 variables were uncorrelated, and indeed the calculated correlation coefficient, 0.074 , is not statistically significant. The facilitation in this example is indicated by the fact that the 
A
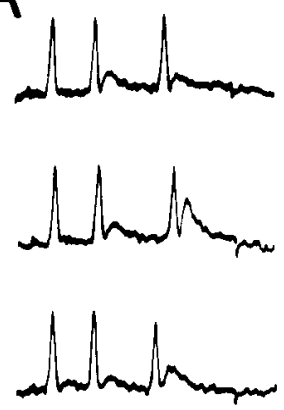

Figure 1. Facilitation of EPSPs mediated by repetitive presynaptic firing. $A$, Five examples of postsynaptic responses (upper traces, Post) evoked by bursts of 3 presynaptic impulses (bottom trace, Pre). The amplitudes of the second and third EPSPs were consistently larger than those of the first EPSPs. $B$, Amplitude histograms of the first (upper, $E P S P_{l}$ ) and second (lower, $\left.E P S P_{2}\right)$ EPSPs obtained from 219 trials in the same experiment as in $A$. The lower histogram is shifted to the right and has a significantly larger mean amplitude $(m)$. The SD's have been corrected for the background noise (variance $=0.053 \mathrm{mV}^{2}$ ).

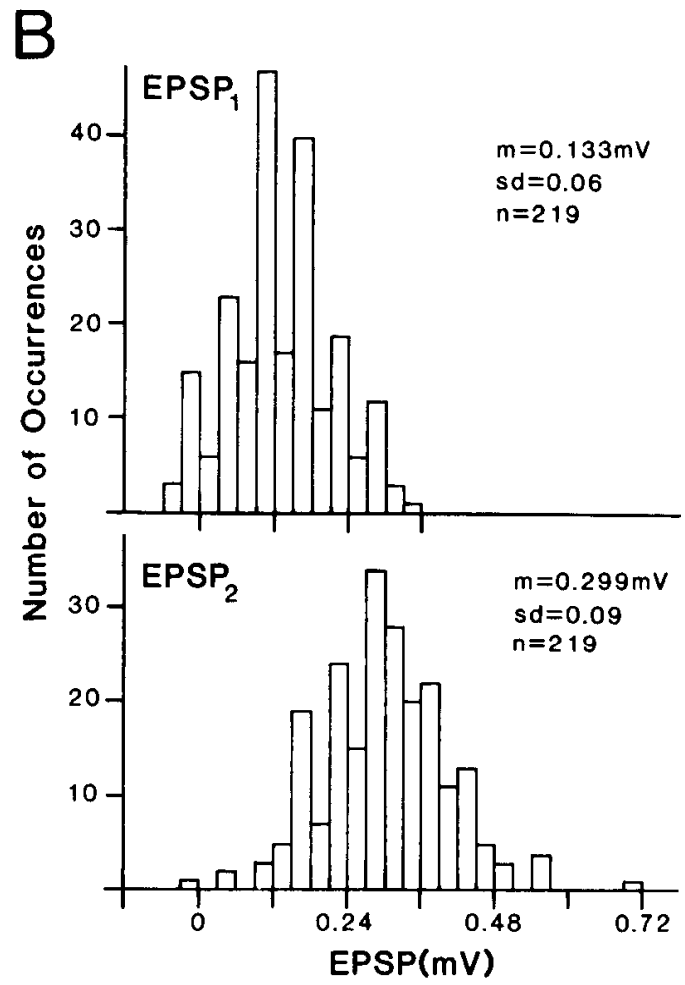

majority of the data points are above the identity line. When all data values in this experiment were ranked according to the amplitudes of the first EPSP and then averaged in successive groups of 22, the average amplitudes of the second EPSP remained constant, ranging from 0.21 to $0.26 \mathrm{mV}$, while those of the first EPSP increased from 0 to $0.3 \mathrm{mV}$ (Fig. $2 B$ ). In other words, the amplitudes of the second EPSPs were independent of those of the first response. The lack of correlation between the 2 EPSPs suggests that facilitation occurs presynaptically and is release independent.

The fluctuations of the third EPSPs were also independent of the variation in the first or second EPSPs or the sum of the 2 , in 8 out of 10 cases analyzed. In the remaining 2 cases, the third EPSP had a slight correlation with EPSP ${ }_{2}$ in 1 and with the sum of the first 2 EPSPs in the other.

A different analysis, based on the assumptions of a binomial release process, also suggests that the facilitation occurred presynaptically. In the binomial model, the CV of an EPSP is a function of $n$ and $p$ and is independent of $q$ (Equation 12). As developed in Materials and Methods, a presynaptic mechanism would result in a smaller CV for the facilitated responses, whereas postsynaptic mechanisms would not alter this parameter. The plot in Figure 3, which summarizes data from 6 experiments, shows that the CV of the second EPSP was always smaller than that of the first. Therefore, if a binomial model for transmitter release pertains in this system, the facilitation of EPSPs mediated by club endings would have a significant presynaptic component.

Is facilitation mediated by an increase of binomial $\mathrm{n}$ ? Although both analyses described above suggest that the facilitation is due to a presynaptic mechanism, the specific variable that has been changed remains unclear. According to the binomial model, the ratios of the mean to variance for the control and facilitated EPSPs should be equal (see equations $11 \mathrm{a}$ and $11 \mathrm{~b}$ ) if the fa- cilitation is associated only with the presynaptic variable $n$. However, this was never the case, as the ratio was consistently greater during facilitation (Table 1), with the possible exception of one experiment (S37A), and, thus, the modified variable is most likely the probability of release. This conclusion would pertain as well if $p, q$, or $n$ varied randomly. In the first 2 cases, the mean to variance ratios would still be expected to be equal, while random variability in $n$, with its mean increasing during facilitation, would result in a decreased ratio.

\section{EPSP fluctuations are independent of presynaptic spike parameters}

Before calculating the binomial variables for the control and test EPSPs, some basic properties of the response fluctuations have to be established, including any possible relation to variations in the associated coupling potentials. The latter analysis will reveal whether the EPSP amplitudes and their fluctuations are dependent upon the extent of presynaptic impulse invasion. Although the EPSP amplitudes were generally small, their fluctuations could be clearly observed by comparing individual records, such as the EPSPs indicated in Figure $4 A$. In this case, the variance of background noise, $9409 \mu \mathrm{V}^{2}$, was smaller than that of the EPSPs and that of the coupling potentials (see Fig. 4, $B$, $C)$. The variances of both components of the postsynaptic potentials were calculated by subtracting the variance of the background noise. For this experiment, the EPSP variance thus calculated, $5720 \mu \mathrm{V}^{2}$, was actually smaller than that of the coupling potentials, $10,191 \mu \mathrm{V}^{2}$. However, the CV of the EPSPs was $20 \%$, which was significantly greater than that of the coupling potentials $(\mathrm{CV}=5 \%)$. That is, the EPSPs exhibited greater fluctuations with respect to their mean amplitudes than did the coupling potentials. For all experiments, the variance of the coupling potentials averaged $9463.5 \pm 7755.0 \mu \mathrm{V}^{2}(n=6)$, which is larger than that of the EPSPs, $3960.4 \pm 1616.2 \mu \mathrm{V}^{2}(n=8)$, but the 

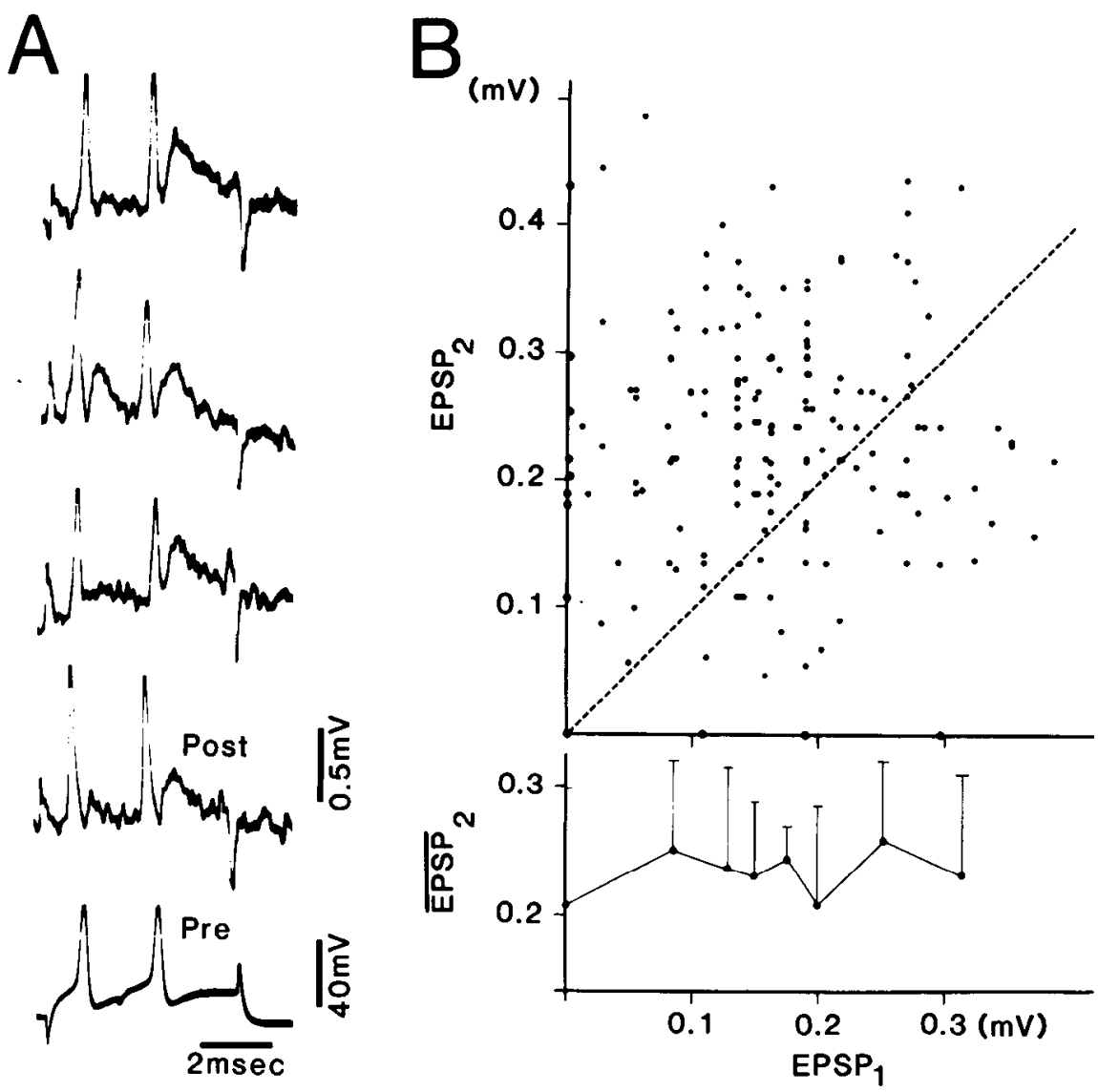

\begin{abstract}
Figure 2. Cross-correlation analysis of control and facilitated EPSPs. $A, U p$ per 4 traces (Post) are M-cell responses evoked by pairs of presynaptic impulses (bottom trace, Pre). Note that the 2 EPSPs fluctuate independently and that in each case the amplitude of the second is greater than that of the first. $B$, Upper graph: plot of the amplitudes of the second EPSPs $\left(\mathrm{EPSP}_{2}\right)$ against those of the first ones $\left(\mathrm{EPSP}_{1}\right)(n=173$ data points). The lack of correlation between the 2 variables is indicated by the random distribution of the data points (correlation coefficient $=0.074$ ). The $45^{\circ}$ line emphasizes the facilitation, since the majority of the points are above this line. Lower graph: same data points ranked according to the amplitudes of EPSP, and averaged in groups of 22 data points. As shown in the lower diagram, the SD's and mean amplitudes of the second EPSPs remained constant as the average amplitudes of EPSP increased.
\end{abstract}

coefficient of variation of the coupling potentials was much smaller than that of EPSPs, $10 \pm 9.2$ versus $40.3 \pm 6.4 \%(n=$ 6).

According to the branch-point hypothesis proposed by Luscher et al. $(1979,1983 a, b)$ to explain PSP fluctuations and PTP, a variable impulse invasion of presynaptic terminals is the main cause of fluctuating transmitter release at individual terminal boutons. Assuming that the amplitudes of coupling potentials accurately reflect the level of impulse invasion in the club endings, an analysis of the correlation between the amplitudes of the coupling potentials and those of the associated EPSPs should reveal whether EPSP amplitudes are dependent upon the magnitude of presynaptic depolarization or, instead, whether the EPSP fluctuations are attributable to the probabilistic nature of the secretion process itself.

The amplitudes of the electrotonic and chemical components evoked by individual presynaptic spikes fluctuated independently. This independence is apparent in the single traces of Figure $5 A$, where, for example, the third coupling potentials have similar amplitudes, but the EPSPs following them do not. When the amplitudes of EPSP 3 were plotted against those of the associated coupling potentials (Fig. $5 B$ ), the scattered distribution of the data points and the low correlation coefficient $(0.03)$ indicated a lack of covariation between the 2 variables. As with the analysis described for Figure $2 B$, the data points in this scatter plot were ranked according to the amplitudes of the coupling potentials and averaged for 8 successive groups of 23 data points each. The average amplitudes of $\mathrm{EPSP}_{3}$ remained constant, while those of the coupling potentials increased from 0.65 to $1.05 \mathrm{mV}$ (Fig. 5B). Such an uncorrelated situation was observed in 14 out of 20 examples analyzed. (The control and facilitated EPSPs from the same fiber were analyzed separately.) The few cases where a positive correlation was found may be the result of variations in the $\mathrm{M}$-cell input resistance, which would cause both components to cofluctuate. The independence

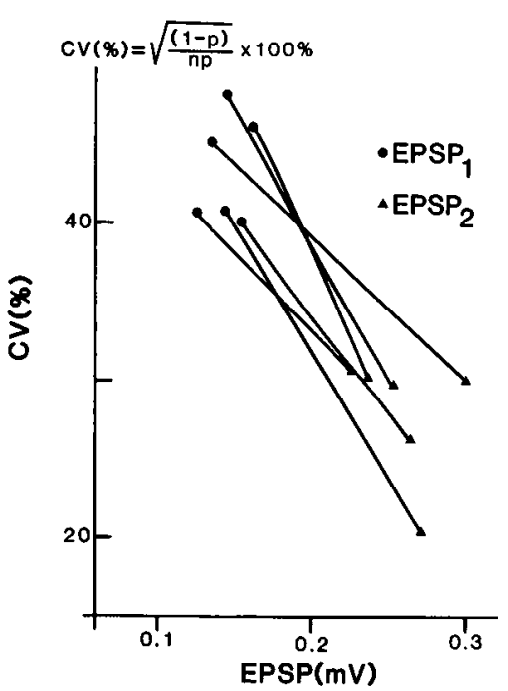

Figure 3. Comparison of the CV of control and facilitated EPSPs. Plot of $\mathrm{CV}$ versus EPSP amplitude for 6 experiments. $\mathrm{CV}$ is equal to the ratio of SD to mean amplitude and can be related to the binomial variables by the indicated equation. Each line connects the CVs calculated for $\operatorname{EPSP}_{1}(\boldsymbol{\bullet})$ and $\operatorname{EPSP}_{2}(\boldsymbol{\Lambda})$ of the same experiment. Facilitation is indicated by the larger amplitudes of the second EPSPs $\left(\mathrm{EPSP}_{2}\right)$. The CVs of the facilitated EPSPs are always smaller than those of the controls. CVs were calculated after correcting for the contribution of background noise to the EPSP amplitude fluctuations (see text). 
Figure 4. Fluctuations of EPSP and coupling potential amplitudes. $A$, Examples of electrotonic coupling potentials $(e)$ and chemically mediated EPSPs (c) evoked by burst of 3 impulses in a single presynaptic fiber (not shown). These traces were collected consecutively, and the fluctuations of the EPSPs following the second coupling potential are apparent. $B$ and $C$, Amplitude histograms of the second coupling potentials and EPSPs, respectively, measured from 182 trials in the experiment of $A$. Although the coupling potential histogram appears broader than the EPSP distributions, the CV of the latter $(20 \%)$ is larger than that of the coupling potentials $(5 \%)$. The indicated SD's of the responses have not been corrected for background noise.

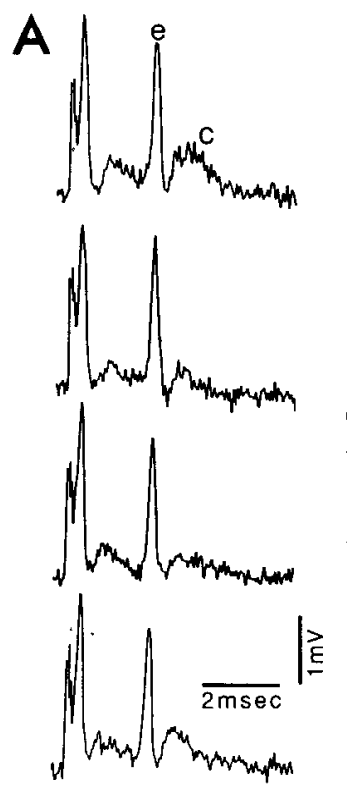

B

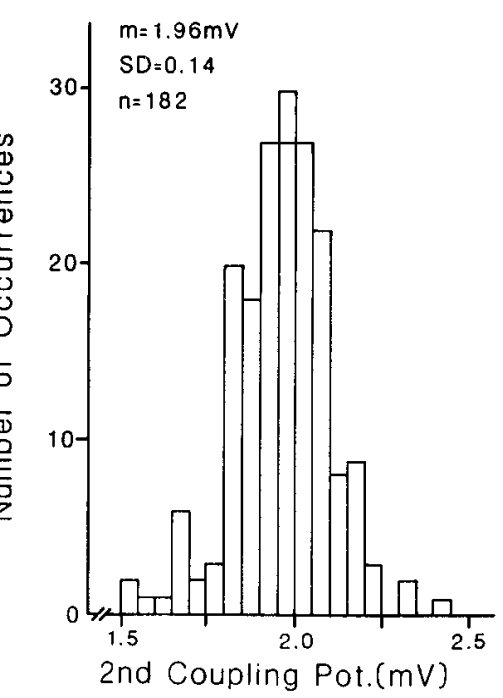

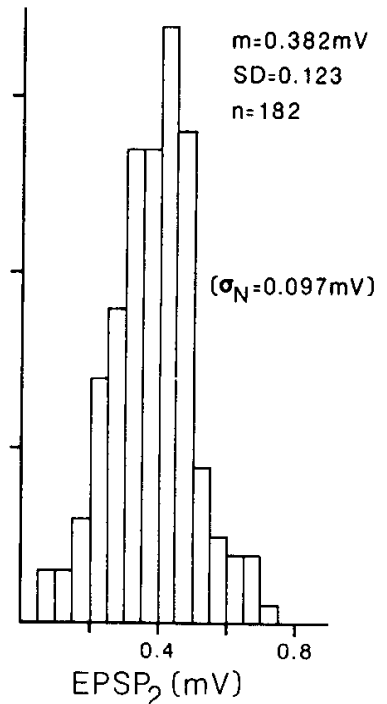

Attempts were first made to analyze the EPSP fluctuations by the procedure developed for central inhibitory synapses, which is based on the binomial assumption and uses the technique of deconvolution to account for the background noise (Korn et al., 1981, 1982). However, the results of this approach were difficult to interpret. In 14 EPSPs thus analyzed, which included both control and facilitated responses, a unique set of binomial parameters was obtained in 7 cases. In 3 of these 7 cases, the deconvoluted binomial parameters provided reasonable fits to the amplitude histograms of facilitated EPSPs, as judged by visual inspection, passed the Kolmogorov-Smirnov test $(p>$ 0.99 ), and $n$ was in the range of $6-15$. The binomial fits in the remaining 4 experiments proved unsatisfactory. Due to the relatively low success rate with this approach, which may be related to a low signal-to-noise ratio (see below), it was not pursued further.

The alternative binomial analysis was based on the added assumption that facilitation is mediated by a uniform increase in the release probability at all active sites, from $p_{1}$ to $p_{2}$, while $n$ and $q$ remain constant since the preceding analyses suggested changes in the latter parameters were unlikely. Given the means and variances of the first and second EPSPs, the 4 unknown binomial parameters can then be calculated (see equations 710). In all the fibers thus analyzed, a unique and biologically meaningful solution (i.e., positive values for $n$ and $p$ ) could be obtained, and the results are summarized in Table 1. They demonstrate that $n$ was generally between 6.0 and 10.8 , that $q$ ranged from 31 to $61 \mu \mathrm{V}$, and that $p_{1}$ varied from 0.29 to 0.44 , with the exception of one experiment, where $n$ was 63 and $p$ equalled 0.07 . Facilitation was associated with an increase in $p$, typically to about $0.51-0.69$. The small quantal size and relatively high noise level excluded the possibility of proving that the individual sets of these variables are the hest fits for the corresponding EPSP histograms. In other words, there may exist other combinations of binomial variables that can as well account for the histograms. Nevertheless, the solutions obtained with this method always provided statistically satisfactory fits to the EPSP amplitude histograms. An example is illustrated in Figure 7, where both the control $(A)$ and facilitated $\operatorname{EPSP}(B)$ distributions change. 


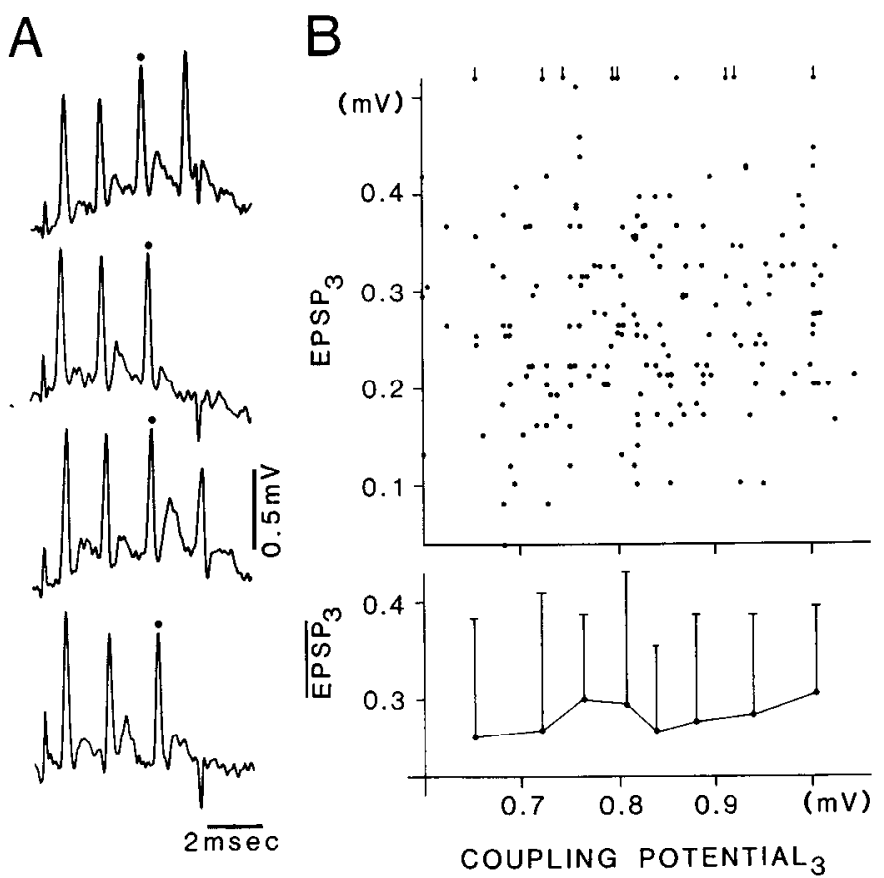

Figure 5. Independent fluctuations of EPSP and coupling potential amplitudes. $A$, Examples of postsynaptic potentials evoked by repetitive firing of a saccular fiber. The third coupling potentials (indicated by the dots) have similar amplitudes in these traces, but they are followed by EPSPs of variable amplitudes. The first upward deflection at the beginning of each trace and the downward deflection near the end are artifacts produced by the on- and offsets of the presynaptic current pulse. The upper graph in $B$ is a plot of amplitudes of the third EPSPs (EPSP $)$ against those of the associated coupling potentials $(n=184)$ and demonstrates that the 2 variables fluctuate independently. Small arrows mark EPSPs $>0.5 \mathrm{mV}$. In the lower graph, the data points were ranked according to the amplitude of the coupling potentials and averaged in groups of 23 points. The average amplitudes and SD's of the EPSP remained constant, while the average amplitudes of the coupling potentials increased from 0.65 to $1.1 \mathrm{mV}$.

are shown for one experiment, in which $p$ increased from 0.29 to 0.51 . Surprisingly, when a similar approach was applied to the third EPSP, the results were not meaningful, as negative $p$ 's and $n$ 's were obtained. In summary, binomial parameters could be estimated by assuming an increase in $p$ during facilitation, and the results provided satisfactory fits to the EPSP histograms. Also, it is not surprising that the deconvolution approach was not successful, because this method is compromised if the SD of background noise is comparable to quantal size (Korn et al., 1982), as was the case at these connections (Table 1).

The effects of random variations in $q, p$, or $n$ on the estimated values can be assessed by referring to equations $(7 a-10 a),(7 b-$ $10 \mathrm{~b})$ and $(7 \mathrm{c}-10 \mathrm{c})$, respectively, and assigning a CV of $25-30 \%$ to each parameter, one at a time. First, direct and indirect analyses of central synapses indicate that $q$ is relatively fixed (Jack et al., 1981a; Korn and Faber, 1987; Korn et al., 1987). Nevertheless, if we assume this parametcr did vary by the indicated amount, the corrected value of $n$ would be unchanged, $p_{1}$ and $p_{2}$ would be about $6-9 \%$ larger than the estimates in Table 1, and $q$ would be $6-8 \%$ smaller. Second, if spatial variation in $p$ has a similar magnitude, the estimated value of $n$ would increase by $6-10 \%$, thereby incrementing this parameter by no more than 1 in 4 of the 5 experiments listed in Table 1 . Also, the probabilities, $p_{1}$ and $p_{2}$, would decrease by $6-8 \%$, and $q$ would be unchanged. Thus, the possible error introduced by assuming $q$
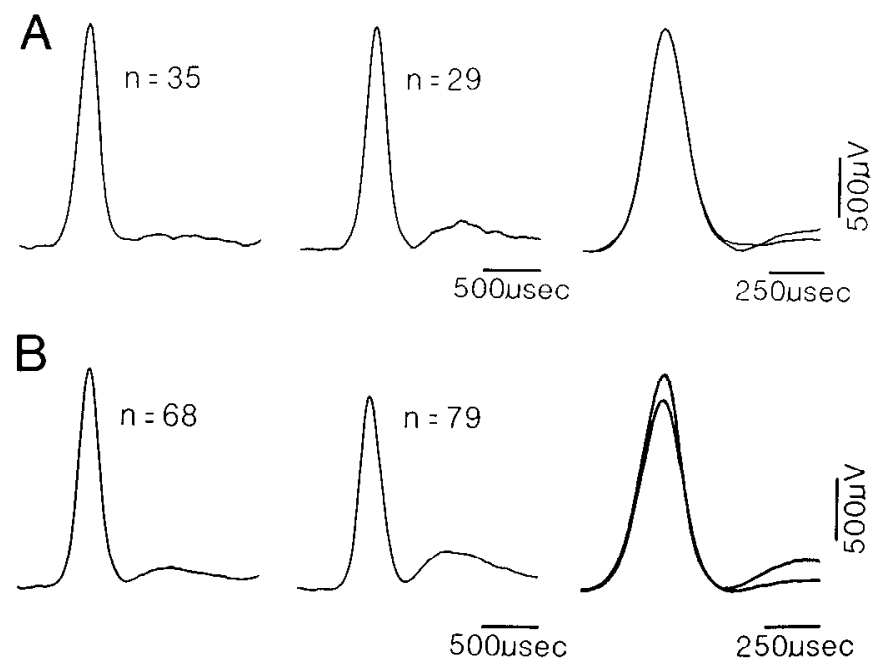

Figure 6. EPSP amplitudes and facilitation are independent of coupling potential duration. $A$, Averaged responses evoked by single presynaptic stimuli. Individual traces from the experiment of Figure 1 were ranked on the basis of the EPSP amplitudes; the first and second records are averages obtained from the bottom and top $25 \%$ of the amplitude histogram, respectively. The 2 are superimposed to the far right at a faster sweep to demonstrate the independence of EPSP amplitude from coupling potential duration. $B$, Records from the same experiment demonstrating that facilitation is not associated with an increase in coupling potential amplitude or duration. First 2 traces are averaged responses evoked by the first and second impulses, respectively. Note the facilitated EPSP in the second record. Since the timing of both presynaptic impulses cxhibited some jitter, data were selceted on the basis of a constant coupling potential onset time. The 2 responses are superimposed at right, with an expanded time base. Note that the 2 coupling potentials have essentially identical time courses but that the amplitude of the second is smaller than that of the first. The label on each trace refers to the number of sweeps used to generate that average.

and $p$ are invariant is quite small. Finally, if there were temporal variations in $n$, again in the range of $25-30 \%$ of the mean, the errors in the estimates of $n$ and $p$ would be larger and dependent upon the value of $n$ itself. For example, corrected values of $n$ would be decreased by about $33-42 \%$ for $n=8, p_{1}$ and $p_{2}$ would be $50-72 \%$ larger, and $q$ would be unaffected. It is difficult to attach significance to the last set of calculations since random variability in the number of release sites has not been described for any system.

\section{Attempts to "unblock" silent connections}

The low incidence of EPSP detection $(<20 \%)$, despite the apparent ultrastructural homogeneity of the club endings, suggests that the complement of chemical synapses in the majority of these terminals is functionally silent. Two facilitatory protocols were tested in attempts to unmask chemical transmission at these junctions. First, the saccular fibers were stimulated repetitively. An example is shown in Figure 8, where bursts of 4 presynaptic impulses were used. The singlc traces in Figure $8 \mathrm{~A}$ show that none of the coupling potentials were followed by EPSPs, and when the signal-to-noise ratio was improved by averaging (Fig. $8 B$ ), there still was no apparent EPSP. Similar negative results were observed in more than 100 cases.

In the second approach, the saccular fiber electrode contained 4-AP for intracellular injection, since this drug has been shown to prolong impulse duration and increase transmitter release either indirectly by blocking voltage-dependent potassium con- 
Figure 7. Binomial description of EPSP fluctuations. A, Amplitude histogram of control, unfacilitated EPSPs together with its binomial fit. The binomial parameters were calculated by assuming an increase in $p$ during facilitation, and the binomial predicted distribution (curve) fit to the actual histogram (bars) was generated from a program incorporating the effects of additive Gaussian noise (Korn et al., 1981, 1982). The binomial parameters used to create the curve were $n=11, p=$ 0.29 , and $q=0.047 \mathrm{mV}$ (see experiment S89F in Table 1). The background noise followed a normal distribution and had an SD of $0.063 \mathrm{mV} . B$, The binomial fit of the facilitated EPSPs obtained from the same experiment as in $A$, with $n=11, p=0.51$, and $q=0.047$ $\mathrm{mV}$. Both fits passed the KolmogorovSmirnov test $(p>0.99)$.

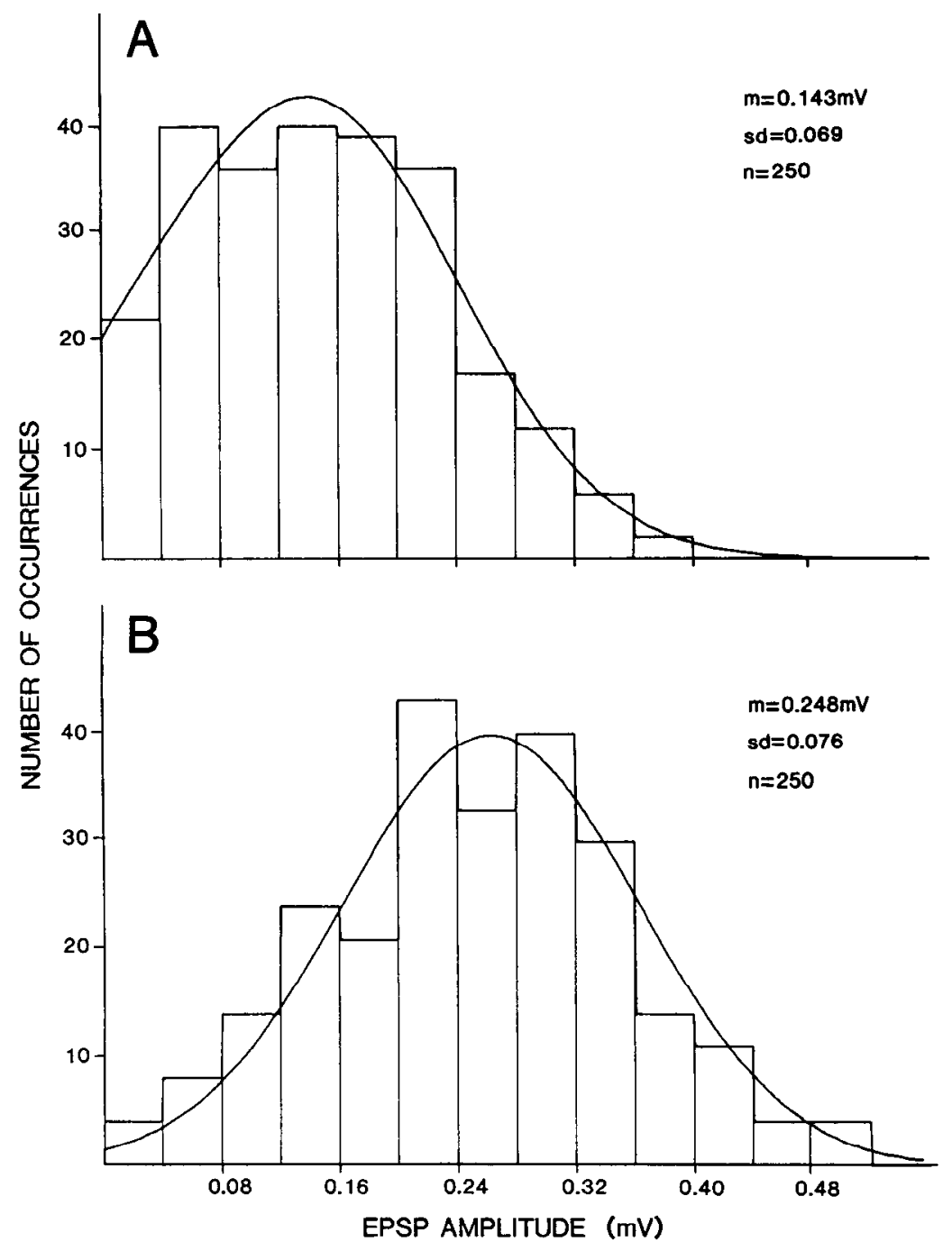

ductances or enhancing calcium influx directly (Yeh et al., 1976; Rogawski and Barker, 1983; for review, see Thesleff, 1980). The major finding with this approach was that, even without iontophoretically injected $4-\mathrm{AP}$, impulses in $56 \%$ of the fibers studied produced EPSPs, which is markedly higher than the occurrence of EPSPs when a $\mathrm{KCl}$ electrode was used $(<20 \%)$. These results were obtained from 15 animals in which the response to every impaled presynaptic fiber, 48 in total, was recorded. And this differential was confirmed in 4 of these 15 fish, when both
$\mathrm{KCl}$ and 4-AP electrodes were tested in each so that variations arising from differences between individual animals could be eliminated. Therefore, 4-AP appears to be effective in activating the silent synaptic connections established by club endings, presumably by increasing presynaptic impulse duration. In a number of these experiments, the unitary EPSP continued to increase in amplitude during the recording session, as illustrated in the example of Figure 9. In this case the EPSP measured within 1 min after the electrode penetration had an amplitude of about

Table 1. Binomial analysis of EPSP fluctuations

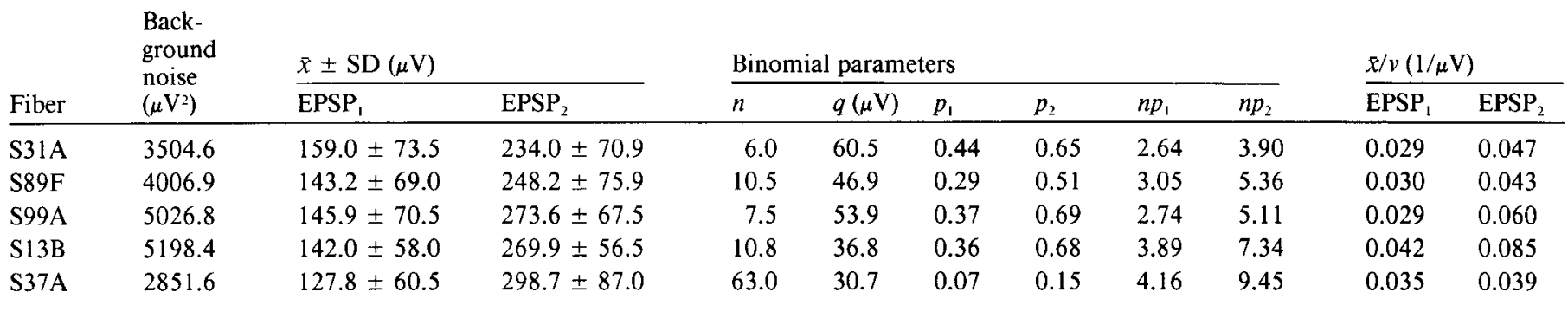




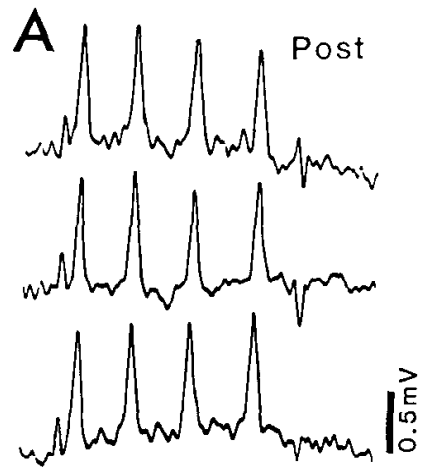

B

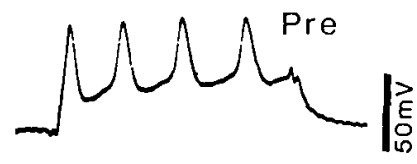

妾

Figure 8. Repetitive firing does not unblock silent synapses. $A$, Postsynaptic responses (upper traces, Post) activated by repetitive presynaptic firing (bottom trace, Pre) exhibit coupling potentials but no chemical components. $B$, Average of 15 postsynaptic traces from the same experiment, with improved signal-to-noise level; note there still is no chemical transmission. The amplitudes of the second, third, and fourth coupling potentials are progressively smaller, due possibly to partial refractoriness of the spike-generating mechanism. Time calibration in $B$ also pertains to $A$.

$80 \mu \mathrm{V}$ (Fig. $9 A$ ) and had grown to more than $200 \mu \mathrm{V}$ with the next 7 min (Fig. $9 B$ ). The coupling potential in Figure $9 B$ is broader than that in $9 A$, indicating that the duration of the terminal action potential also increased during the same time period.

In almost all the experiments where chemical transmission was demonstrated with a 4-AP electrode ( $n=27$ fibers), an EPSP was observed within $1 \mathrm{~min}$ after electrode penetration. This was unexpected since the presynaptic recording site was typically $700 \mu \mathrm{m}$ away from the club endings. However, careful inspection showed that the duration of presynaptic impulses could be increased within $30 \mathrm{sec}$ after the fiber had been penetrated, as illustrated in the example of Figure 10. In another 10 experiments in which the quality of presynaptic recording was satisfactory (spike amplitudes $>50 \mathrm{mV}$ ), measurable increases in spike duration occurred over time periods of 30-60 sec. If such rapid spike broadening is conducted to the club endings electrically, there should be a similar increase in duration of the coupling potentials at the same timc. In confirmation, the average half-width of the coupling potentials recorded within the first 2 min after penetrating afferent fibers with 4-AP electrodes was $320 \pm 92 \mu \mathrm{sec}(n=11)$, which is significantly longer than that found in control experiments with $\mathrm{KCl}$ electrodes $(242 \pm 16 \mu \mathrm{sec}, n=9$; the sample sizes are small because only data from experiments recorded with a cut-off frequency of $10 \mathrm{kHz}$ were used). However, while 4-AP increased the occurrence of EPSPs and injections of the drug enhanced EPSPs already present, such injections had no effect on the remaining $44 \%$ of the connections that were still silent. Finally, when the data obtained with $\mathrm{KCl}$ and 4-AP electrodes were treated separately, coupling potential durations were the same for transmitting and silent connections.

A second potassium blocker, Cs, was also tested, but the results were less consistent. With $\mathrm{Cs}$ electrodes, the percentage
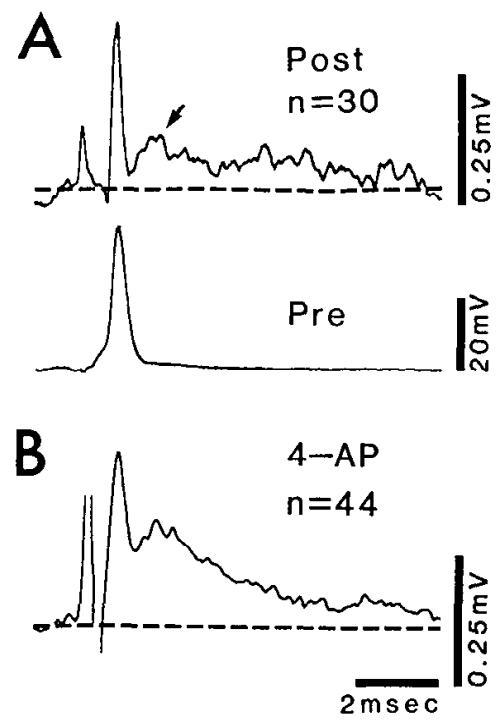

Figure 9. Facilitatory effect of 4-AP on chemical transmission mediated by club endings. $A$, Coupling potential and EPSP (arrow in the upper trace, Post) produced by single impulses in a saccular fiber (lower trace, Pre). Both records are the average of 30 sweeps and were collected within $1 \mathrm{~min}$ after the 4-AP-containing electrode penetrated the fiber. The presynaptic impulse was activated by a short current pulse, as indicated by the upward- and downward-going artifacts preceding the coupling potential. The dashed line indicates the M-cell resting membrane potential. $B$, In the same experiment, the mean EPSP $(n=44)$ collected $8 \mathrm{~min}$ after penetration was enhanced and prolonged by 4-AP. The artifact due to presynaptic current injection became larger, because the presynaptic recording condition had deteriorated and a larger current pulse was needed to activate action potentials. The effect of 4-AP on presynaptic impulse duration is clearly indicated by the broader coupling potential.

of the chemically active synapses was $25 \%$ in the control period, i.e., within $2 \mathrm{~min}$ after electrode penetration and before $\mathrm{Cs}$ injection, and this value increased to only $29 \%$ after Cs injection. The half-width of the coupling potentials in the presence of $\mathrm{Cs}$ was $254 \pm 34 \mu \mathrm{sec}(n=20)$ in the control period, increasing to $302 \pm 48 \mu \mathrm{sec}(n=17)$ after one injection session. Thus, although $\mathrm{Cs}$ injections could produce a broadening of presynaptic impulse comparable to that seen in the presence of 4-AP, they were much less effective in activating the silent terminals. The only noticeable difference between the effects of the 2 agents on presynaptic impulses is that when the same degree of impulse broadening was achieved by both drugs, 4-AP more effectively eliminated the afterhyperpolarization following individual spikes than did Cs.

Quantitative relationship between coupling potential durations and transmitter output. The mixed configuration of the club ending synapses provides a unique opportunity to establish a quantitative relationship between presynaptic impulse duration and transmitter output for vertebrate central synapses. An example of this relationship is illustrated in Figure 11. In this experiment, the control EPSP, amplitude and the associated coupling potential half-width were $36 \mu \mathrm{V}$ and $248 \mu \mathrm{sec}$, respectively (Fig. $11 A I$ ), and indeed there was no clear EPSP. After $3 \mathrm{Cs}$ injection sessions, the EPSP amplitude was increased to $396 \mu \mathrm{V}$ and the half-width of the coupling potentials rose to $352 \mu \mathrm{sec}$ (Fig. 11A2). The input-output relationship plotted in Figure $11 B$ indicates that after the first injection an increase in spike duration of about $75 \mu \mathrm{sec}$ was associated with the emergence of a clear EPSP, about $160 \mu \mathrm{V}$ in amplitude. The overall 


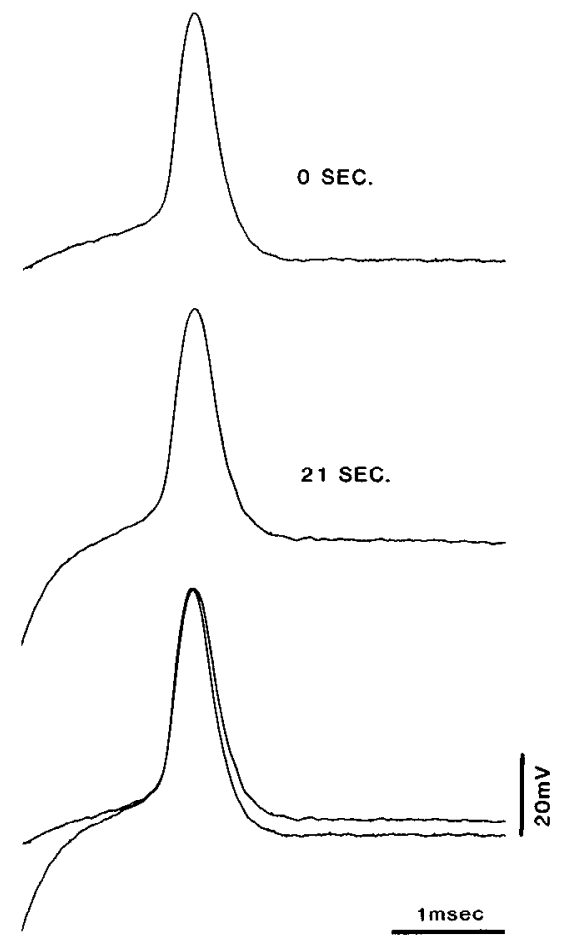

Figure 10. Rapid action of 4-AP on presynaptic impulse duration. The action potential shown in the upper trace is the first one recorded after a 4-AP-containing electrode penetrated a saccular fiber. The middle trace shows the 16th action potential in the same experiment, obtained 21 $\mathrm{sec}$ after penetration. The longer spike duration is further demonstrated by superimposing the 2 traces (bottom), with their initial rising phases and peaks being aligned. slope of the plot relating response amplitude to spike duration is $7.1 \mathrm{mV} / \mathrm{msec}$. In 4 other experiments, in which the broadening of the presynaptic impulses developed slowly enough to allow a similar detailed analysis, the slope ranged from 1.2 to $5.5 \mathrm{mV} /$ $\mathrm{msec}$, which is comparable to the sensitivities of the lower and intermediate regions of the input-output curves obtained from the squid giant synapse (6-7 mV/msec; Llinás et al., 1981) and Aplysia sensory-motor synapses $(5 \mathrm{mV} / \mathrm{msec}$; Hochner et al., 1986). Also, the EPSP rate of rise increased when impulse broadening was associated with potentiation, as indicated in the superimposed traces in the inset of Figure $11 B$. In that example, the rate of rise is $0.97 \mathrm{mV} / \mathrm{msec}$ for the largest EPSP and 0.582 and $0.346 \mathrm{mV} / \mathrm{msec}$ for the second and third largest ones, respectively. Similar observations were made in another experiment, in which the rate of rise increased from 0.86 to $1.31 \mathrm{mV} /$ msec as the EPSP amplitude increased from 312 to $514 \mu \mathrm{V}$.

\section{Discussion}

The primary findings reported here concerning excitatory synaptic transmission between club endings and the $\mathrm{M}$-cell are that (1) the augmentation of EPSP amplitudes recorded in the Mcell during repetitive presynaptic stimulation is due to facilitated transmitter release, which, on the basis of a binomial model, appears to be associated with an increase in the probability of release; (2) EPSP fluctuations and facilitation are not correlated with variations in the magnitude and duration of the terminal action potential; (3) the binomial variable $n$ is significantly greater than 1 at these single-ended connections; and (4) because of the brief nature of the presynaptic impulse, these junctions normally operate at the low end of the input-output relation, such that small increases in spike duration can unblock "silent connections" and produce marked increases in EPSP amplitudes.

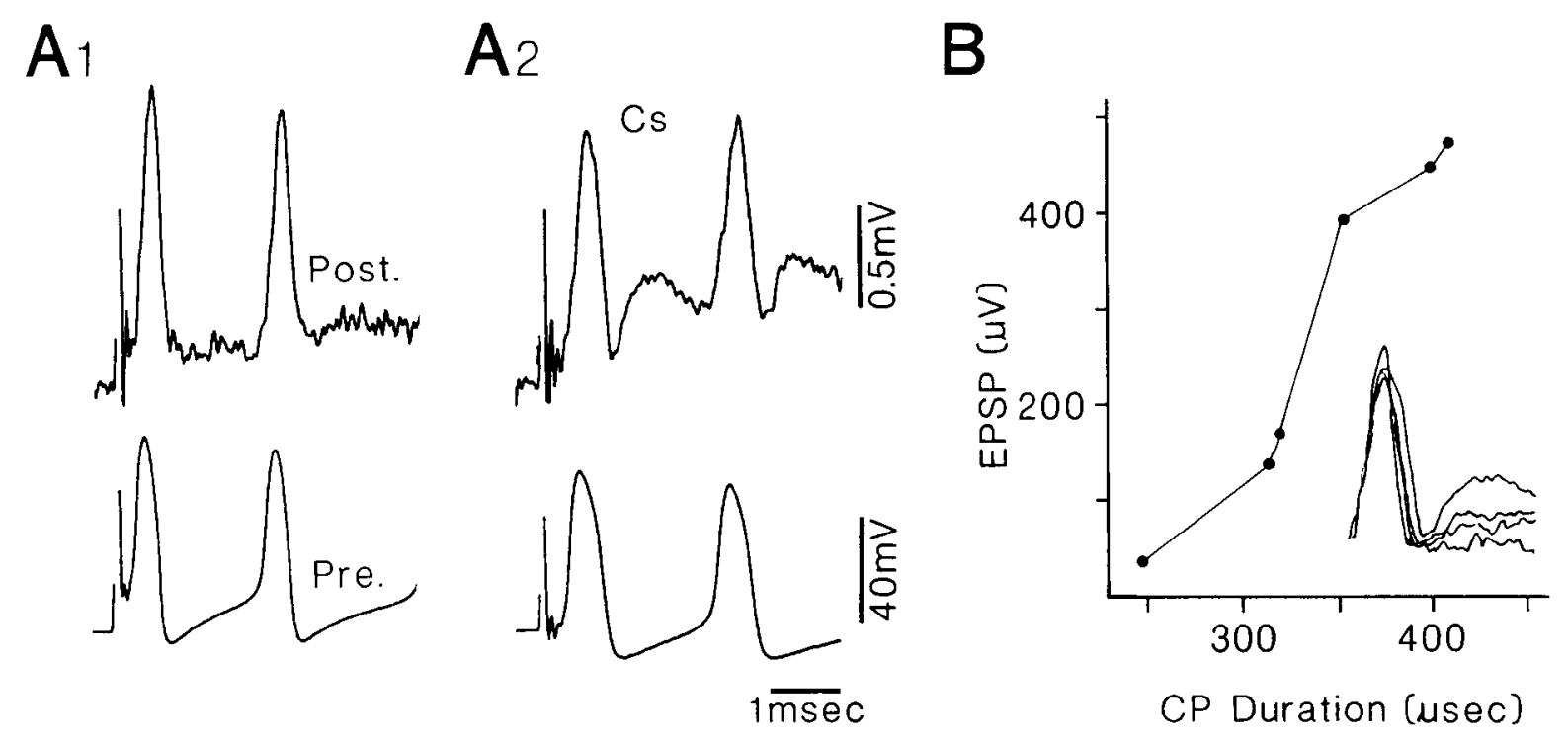

Figure 11. Quantitative relationship between coupling potential duration and EPSP amplitude. The impulse broadening observed in this example was obtained by $\mathrm{Cs}$ injection into the presynaptic fiber. A1, Average traces of pre- and postsynaptic recordings $(n=9)$ collected before Cs injection. The half-width of the first coupling potential was $244 \mu \mathrm{sec}$, and there was virtually no EPSP following it. The average is obtained from the traces whose second impulses have the same latencies. A2, Average of 17 traces after 3 periods of Cs injection. Note the broader coupling potentials (halfwidth $=356 \mu \mathrm{sec}$ ) and significantly larger EPSP amplitudes. Cs was injected by subthreshould depolarizing current pulses repeated at a rate of 20 $\mathrm{Hz}$ with an $80 \%$ duty cycle. $B$, Plot of coupling potential $(C P)$ half-widths and EPSP amplitudes from the experiment illustrated in $A 1$ and $A 2$, respectively, but are averages based on larger sample sizes $(n=25-54)$. The slope in the steepest region is $7.1 \mathrm{mV} / \mathrm{msec}$. Inset, Superimposed traces of the first coupling potentials and associated EPSPs. The smallest and largest EPSP have been shown in $A 1$ and $A 2$, the intermediate traces correspond to the data collected following the first and second Cs injections. 


\section{Paired-pulse facilitation}

Mechanisms. We have shown that the paired-pulse facilitation mediated by the club ending synapses most likely occurs presynaptically and, on the basis of a simple binomial model, is associated with an increase in $p$. This conclusion is similar to that reached concerning the PTP observed at Ia-motoneuron synapses (Hirst et al., 1981) and the crayfish ncuromuscular junction (Zucker, 1973). However, the behavior of these other connections is different from that of the club ending synapses, which do not exhibit either PTP or the frequency facilitation seen during maintained repetitive stimulation of Ia fibers and the crayfish motor nerve (Curtis and Eccles, 1960; Wojtowicz and Atwood, 1985).

The branch-point failure hypothesis for PTP at the Ia to motoneuron synapses (Luscher et al., 1979, 1983a, b; but see Jack et al., 1981a) assumes that release is dependent upon the active invasion of the presynaptic terminal and that, while individual boutons always release transmitter when actively invaded by an impulse, the EPSP fluctuates because of intermittent conduction failures at axonal branch points. From that perspective, PTP is viewed as resulting from an improved safety factor for impulse conduction at such branch points. Numerous results and analyses provide compelling arguments against this explanation of PSP fluctuations (Jack et al., 1981; Korn et al., 1982; Korn and Faber, 1987) and PTP (Hirst et al., 1981). Certainly, the present findings cannot be explained by the branch-point failure hypothesis, since most (97\%) of the saccular fibers terminate on the M-cell as single club endings (Lin et al., 1983) and the EPSP amplitudes were uncorrelated with the magnitudes and durations of the associated coupling potentials for both control and facilitated responses. In fact, the amplitudes of the second coupling potentials were generally smaller than those of the first ones (e.g., Figs. 1 and 6). In conclusion, then, the mechanisms responsible for the fluctuations of EPSP amplitude are more likely related to the secretion process itself rather than to a variable presynaptic depolarization. This lack of a correlation also suggests that the spikes may be significantly larger than the potential that fully activates the calcium channels, while the existence of facilitation indicates that the secretion process itself is not saturated.

The present results are consistent with the general notion that the release probability is the binomial variable most subject to modification during instances of short-term plasticity (Korn et al., 1984; Korn and Faber, 1987). Conclusive evidence for an increase in binomial $p$ during facilitation was demonstrated at the crayfish ncuromuscular junction (Zucker, 1973), and variations in this parameter best accounted for the effects of changes in extracellular calcium concentration at the mammalian neuromuscular junction (Christensen and Martin, 1970) and cultured spinal synapses (Nelson et al., 1983). Also, frequencydependent depression of the M-cell inhibitory synapses was clearly associated with a decrease in $p$ (Korn et al., 1984). Given the linited understanding of molecular mechanisms of exocytosis, biochemical or structural correlates of the probability of quantal release remain unknown. Certainly, a great deal of evidence suggests it is related to presynaptic calcium activity at vesicle release sites, with short-term facilitation generally being linked to residual increases in intracellular calcium concentration (Katz and Miledi, 1968; cf. model by Zucker and Stockbridge, 1983). These concepts are partly based on observations that at the crayfish neuromuscular junction facilitation is in- dependent of presynaptic spike parameters (Zucker, 1974; Zucker and Lara-Estrella, 1979). Our findings that coupling potential amplitude and duration do not change during facilitation are consistent with such conclusions and extend them, for the first time, to vertebrate central synapses.

Physiological significance of facilitation. The magnitude of facilitation mediated by the club ending synapses, $99 \%$ in avcrage, is quitc strong comparcd with othcr CNS junctions. For example, the maximal paired-pulse facilitation observed at Iamotoneuron and corticospinal synapses was 30\% (Hirst et al., 1981 ) and 60\% (Muir and Porter, 1973), respectively. This diversity suggests that the release process is tuned differently (see discussion in Korn and Faber, 1987) at individual synapses, possibly according to the integration requirements of each systern. In the case of club endings, the saccular fibers fire at frequencies equal to or twice that of applied pure tones (Furukawa and Ishii, 1967). The best frequencies for the large-diameter saccular fibers, which presumably terminate on the M-cell as club endings (Furukawa and Ishii 1967), were between 300 to $800 \mathrm{~Hz}$ (Fay and Olsho, 1979), and the interspike intervals would thus be in the range of $1.25-3 \mathrm{msec}$. Therefore, the facilitated EPSPs studied here occur within a physiological range of presynaptic firing rates and probably serve the function of amplifying high-frequency signals. However, this amplification is restricted to a short-term basis, since the junctions did not exhibit PTP or frequency facilitation.

\section{EPSP fluctuations and binomial variables}

Since the correlation analysis of paired EPSP amplitudes and comparison of the CVs of control and facilitated responses suggested that facilitation occurred presynaptically and could not be associated with a change in binomial $n$ alone, we tested the hypothesis that it was instead due to an increase in $p$. With the assumption of a simple binomial model, biologically meaningful (real) parameters were obtained for all the connections analyzed, i.e., $0<p<1$ and $n>0$. However, while a simple binomial model described the release process for the first and second EPSPs, this approach yielded negative values for $n$ and $p$ when applied to the facilitation associated with $\operatorname{EPSP}_{3}$. This failure suggests that the fluctuations of EPSP $_{3}$ deviate from a simple binomial model, or the facilitation involved changes in more than one parameter, which could be the case if some opposing depression occurs during the third EPSP. It also confirms that this analysis can sometimes discriminate between conditions that do and do not conform to the assumptions of this model.

The ranges of the derived binomial variables $-n, p$, and $q-$ were comparable to the corresponding values extracted from other central synaptic connections. (Since the possible errors caused by ignoring variations in these parameters were generally small, and there is no independent method to determine their magnitudes, the uncorrected values in Table 1 are discussed here.) The quantal size, 36-60 $\mu \mathrm{V}$, is small compared with the value of $100 \mu \mathrm{V}$ estimated for Ia-motoneuron junctions and the synapses between cultured CNS neurons (Jack et al., 1981 a; Nelson et al., 1983). However, when this parameter is converted into the underlying conductance change, the value of $3 \mathrm{nS}$ derived for the club endings is slightly larger than the corresponding estimates for other excitatory CNS junctions, i.e., $1-1.5 \mathrm{nS}$ (Finkel and Redman, 1983; Nelson et al., 1986). The range of $p$, from 0.29 to 0.44 , is also similar to that obtained from a central inhibitory synapse (Korn et al., 1981, 1982).

The calculated values of binomial $n$ varied from 6 to 11 , 
which is lower than, but overlaps with, the number of morphologically defined active zones in a club ending - up to 38 (Kohno and Noguchi, 1986). Since the club endings studied physiologically were not examined at the ultrastructural level, the precise relationship, if any, between physiological $n$ and the number of active zones remains a matter of speculation. However, our findings are consistent with the earlier conclusion that active zones in the synaptic terminal are the elementary release units (Korn et al., 1982; Triller and Korn, 1982) and confirms the generalization of that notion to terminals with multiple active zones (Korn and Faber, 1987). A similar generalization was proposed on the basis of studies of muscle afferent inputs to dorsal spinocerebellar tract (DSCT) neurons in the cat, where single afferent neurons have multiple terminals on the DSCT cells, some of which may be giant boutons with multiple active zones (Tracey and Walmsley, 1984; Walmsley et al., 1985). However, since there was no combined morphophysiological study at the level of individual afferent units, it is unclear whether the connections studied physiologically contained such giant ooutons, and the results thus did not warrant the authors' conclusion. Different findings were obtained from cultured rat spinal neurons, where there is an average of 2 active zones per bouton (Neale et al., 1983), but it has been suggested that each ending is a quantal unit (Pun et al., 1986), a notion not supportcd by our observations. In fact, we specifically asked whether the fluctuations of EPSPs mediated by single club endings could be accounted for by a binomial model with $n=1$. Under this assumption, values for binomial $p$ and $q$ were calculated from the mean and variance of the EPSPs, but then the predicted EPSP amplitude distributions incorporating the measured background noise provided statistically inadequate fits of the original histograms in 9 of 12 cases (Lin, 1986). Thus, the idea that $n=$ 1 for a bouton with multiple active zones can be rejected for the club endings in favor of the hypothesis that each such site releases transmitter independently.

\section{Modulation of silent synaptic connections}

Two sets of observations lead us to propose that, while impulses in the majority of the club endings do not evoke detectable EPSPs, the blockade can be reversed. First, electron microscopic studies of club endings demonstrated that all of them have the structural correlates of chemical synapses, suggesting that the machinery for chemical transmission is present (Nakajima, 1974; Kohno and Noguchi, 1986; Tuttle et al., 1986). Second, and most importantly, the demonstration that intra-axonal injection of 4-AP could effectively increase the occurrence of chemical transmission provided strong support for the reversibility of these silent connections.

Although spike broadening clearly enhanced synaptic transmission at the club ending synapses on the M-cell, the specific mechanisms underlying the blockade under physiological conditions remain unclear. In the study where Ia-motoneuron transmission was enhanced by 4 -AP, the proposed activation mechanism was based on the drug's known pharmacological effect; i.e., broadening of presynaptic impulses (Jack et al., 1981b). If the only effect of 4-AP is to block potassium conductance, the voltage-sensitive calcium influx in the presynaptic terminal may be increased significantly by impulse prologation and may be the critical element in the control of transmitter release by these terminals. Our findings are consistent with this line of interpretation, in that we demonstrated a direct correlation between presynaptic impulse broadening and activation of the silent syn- aptic connections with 4-AP. However, this may be an overly simplistic interpretation because Cs injections were not as effective as 4-AP when the level of impulse broadening was the same for both agents. Thus, 4-AP may have effects other than blocking potassium conductance (Muller, 1986), or the 2 compounds may preferentially block different potassium conductances. Finally, we also encountered fibers in which 4-AP or Cs could effectively increase impulse duration without inducing transmission. Therefore, in some club endings, other factors, such as the absence of functioning calcium channels, may be responsible for the appearance of silent contacts.

Input-output function for club ending synapses. With 4-APor Cs-filled electrodes, we were able to obtain a quantitative relationship between transmitter output and the half-width of coupling potentials, which provide a good measure of the waveform of the terminal action potential. This, to our knowledge, is the first such description generated for vertebrate central synapses. The slope of this input-output relationship varied over a wide range, the high end of which overlapped with that obtained from the squid giant synapse and Aplysia sensorimotor synapses (Llinás et al., 1981; Hochner et al., 1986). The large variation of the input-output slope may be due to heterogeneity of the club endings or to factors that were not controlled under the present cxpcrimental conditions.

In the squid giant synapse, EPSPs evoked by short-duration presynaptic pulses $(<1.5 \mathrm{msec}$ ) have a slower rate of rise than those evoked by broader pulses, and the slow rise time is presumably related to the small "off' calcium influx that starts after the presynaptic pulse is terminated (Llinás et al., 1981). The finding that the EPSP rate of rise increased following lengthening of the coupling potentials and the normally extremely short duration of saccular fiber impulses $(<0.5 \mathrm{msec})$ are consistent with this observation. Thus, we suggest that club endings cperate in the low end of their input-output curve under control conditions. Presumably, the exocytoses mediated by multiple active zones were somewhat asynchronous in control conditions but became more synchronous when impulse duration was increased by 4-AP or Cs. Alternatively, the acceleration in the rate of rise might be attributed to unknown mechanisms that are independent of the increased calcium influx associated with impulse broadening (Hochner et al., 1986).

\section{References}

Brown, T. H., D. H. Perkel, and M. W. Feldman (1976) Evoked neurotransmitter release: Statistical effects of nonuniformity and nonstationarity. Proc. Natl. Acad. Sci. USA 73: 2913-2917.

Christensen, B. N., and A. R. Martin (1970) Estimates of probability of transmitter release at the mammalian neuromuscular junction. $\mathrm{J}$. Physiol. (Lond.) 210: 933-945.

Curtis, D. R., and J. C. Eccles (1960) Synaptic action during and after repetitive stimulation. J. Physiol. (Lond.) 150: 374-398.

Dostrovsky, J. O., J. Millar, and P. D. Wall (1976) The immediate shift of afferent drive of dorsal column nucleus cells following deafferentation: A comparison of acute and chronic deafferentation in gracile nucleus and spinal cord. Exp. Neurol. 52: 480-495.

Fay, R. R., and L. W. Olsho (1979) Discharge patterns of lagenar and saccular neurons of goldfish eighth nerve: Displacement sensitivity and directional characteristics. Comp. Biochem. Physiol. 62A:377386.

Finkel, A. S., and S. J. Redman (1983) The synaptic current evoked in cat spinal motoneurones by impulses in single group Ia axons. J. Physiol. (Lond.) 342: 615-632.

Furukawa, T., and Y. Ishii (1967) Neurophysiological studies of hearing in goldfish. J. Neurophysiol. 30: 1377-1402.

Henneman, E., H.-R. Luscher, and J. Mathis (1984) Simultaneously 
active and inactive synapes of single Ia fibers on cat spinal motoneurons. J. Physiol. (Lond.) 352: 147-161.

Hirst, G. D. S., S. J. Redman, and K. Wong (1981) Post-tetanic potentiation and facilitation of synaptic potentials evoked in cat spinal motoneurones. J. Physiol. (Lond.) 321: 97-109.

Hochner, B., M. Klein, S. Schacher, and E. R. Kandel (1986) Additional component in the cellular mechanism of presynaptic facilitation contributes to behavioral dishabituation in Aplysia. Proc. Natl. Acad Sci. 83: 8794-8798.

Jack, J. J. B., S. J. Redman, and K. Wong (1981a) The components of synaptic potentials evoked in cat spinal motoneurones by impulses in single group la afferents. J. Physiol. (Lond.) 321: 65-96.

Jack, J. J. B., S. J. Redman, and K. Wong (1981b) Modifications to synaptic transmission at group la synapses on cat spinal motoneurones by 4-aminopyridine. J. Physiol. (Lond.) 321: 111-126.

Katz, B., and R. Miled (1968) The role of calcium in neuromuscular facilitation. J. Physiol. (Lond.) 195: 481-492.

Kohno, K., and N. Noguchi (1986) Large myelinated club endings on the Mauthner cell in the goldfish: A study with thin sectioning and freeze-fracturing. Anat. Embryol. 173: 361-3.70.

Korn, H., and D. S. Faber (1987) Regulation and significance of probabilistic release mechanisms at central synapses. In Synaptic Function, G. M. Edelman, W. E. Gall, and W. M. Cowan, eds., pp. 57-108, Wiley, New York.

Korn, H., A. Triller, A. Mallet, and D. S. Faber (1981) Fluctuating responses at a central synapse: $n$ of binomial fit predicts number of stained presynaptic boutons. Science 213: 898-901.

Korn, H., A. Mallet, A. Triller, and D. S. Faber (1982) Transmission at a central inhibitory synapse. II. Quantal description of release, with a physical correlate of binomial $n$. J. Neurophysiol. 48: 679-707.

Korn, H., D. S. Faber, Y. Brunod, and A. Triller (1984) Regulation of efficacy at central synapses. J. Neurosci. 4: 125-130.

Korn, H., Y. Burnod, and D. S. Faber (1987) Spontaneous quantal currents in a central neuron match predictions from binomial analysis of evoked responses. Proc. Natl. Acad. Sci. USA 84: 5981-5985.

Lin, J.-W. (1986) Physiology and morphology of identified mixed excitatory synapses on the goldfish Mauthner cell. Ph.D. thesis, SUNY at Buffalo, Buffalo, NY.

Lin, J.-W., and D. S. Faber (1988) Synaptic transmission mediated by single club endings on the goldfish Mauthner cell. I. Characteristics of electrotonic and chemical postsynaptic potentials. J. Neurosci. 8: 1302-1312.

I.in, J.-W., D. S Faber, and M. R. Wood (1983) Organized projection of the goldfish saccular nerve onto the Mauthner cell lateral dendrite. Brain Res. 274: 319-324.

Llinás, R., I. Z. Steinberg, and K. Walton (1981) Relationship between presynaptic calcium current and postsynaptic potential in squid giant synapse. Biophys. J. 33: 323-352.

Lomo, T. (1971) Potentiation of monosynaptic EPSPs in the perforant path-dentate granule cell synapse. Exp. Brain Res. 12: 46-63.

Luscher, H.-R., P. Ruenzel, and E. Henneman (1979) How the size of motoneurones determines their susceptibility to discharge. Nature 282: 859-861.

Luscher, H.-R., P. Ruenzel, and E. Henneman (1983a) Composite EPSPs in motoneurons of different sizes before and during PTP: Implications for transmission failure and its relief in Ia projections. $J$. Neurophysiol. 49: 269-289.

Luscher, H.-R., P. W. Ruenzel, and E. Henneman (1983b) Effects of impulse frequency, PTP and temperature on responses elicited in large populations of motoneurons by impulses in single la-fibers. J. Neurophysiol. 50: 1045-1058.

Millar, J., A. I. Basbaum, and P. D. Wall (1976) Restructuring of the somatotopic map and appearance of abnormal neuronal activity in the gracile nucleus after partial deafferentation. Exp. Neurol. 50: 658672 .

McLachlan, E. M. (1978) The statistics of transmitter release at chemical synapses. Int. Rev. Physiol. 17: 49-117.

Muir, R. B., and R. Porter (1973) The effect of preceding stimulus on temporal facilitation at corticomotoneuronal synapses. J. Physiol. (Lond.) 228: 749-763.
Muller, D. (1986) Potentiation by 4-aminopyridine of quantal acetylcholine release at the torpedo nerve-electroplaque junction. J. Physiol. 379: 479-493.

Nakajima, Y. (1974) Fine structurc of the synaptic endings on the Mauthner cell of the goldfish. J. Comp. Neurol. 156: 375-402.

Nakajima, Y., and K. Kohno (1978) Fine structure of the Mauthner cell: Synaptic topography and comparative study. In Neurobiology of the Mauthner Cell, D. S. Faber and H. Korn, eds., pp. 133-166, Raven, New York.

Neale, E. A., P. G. Nelson, R. L. Macdonald, C. N. Christian, and L. M. Bowers (1983) Synaptic interactions between mammalian central neurons in cell culture. III. Morphological correlates of quantal synaptic transmission. J. Neurophysiol. 49: 1459-1468.

Nelson, P. G., K. C. Marshall, R. Y. K. Pun, C. N. Christian, W. H. Sheriff, Jr., R. L. Macdonald, and E. A. Neale (1983) Synaptic interactions between mammalian central neurons in cell culture. II. Quantal analysis of EPSPs. J. Neurophysiol. 49: 1442-1458.

Nelson, P. G., R. Y. K. Pun, and G. L. Westbrook (1986) Synaptic excitation in cultures of mouse spinal cord neurons: Receptor pharmacology and behaviour of synaptic current. J. Physiol. (Lond.) 372: 169-190.

Nelson, S. G., T. C. Collatos, A. Niechaj, and L. M. Mendell (1979) Immediate increase in Ia-motoneuron synaptic transmission caudal to spinal cord transection. J. Neurophysiol. 42: 655-664.

Porter, R. (1970) Early facilitation at corticomotoneuronal synapses. J. Physiol. (Lond.) 207: 733-745.

Pun, R. Y. K., E. A. Neale, P. B. Guthrie, and P. G. Nelson (1986) Active and inactive central synapses in cell culture. J. Neurophysiol. 56: $1242-1256$.

Redman, S., and B. Walmsley (1983) Amplitude fluctuations in synaptic potentials evoked in cat spinal motoneurones at identified group Ia synapses. J. Physiol. (Lond.) 343: 135-145.

Rogawski, M. A., and J. L. Barker (1983) Effects of 4-aminopyridine on calcium action potentials and calcium current under voltage clamp in spinal neurons. Brain Res. 280: 180-185.

Thesleff, S. (1980) Aminopyridines and synaptic transmission. Neuroscience 5: 1413-1419.

Tracey, D. J., and B. Walmsley (1984) Synaptic input from identified muscle afferents to neurones of the dorsal spinocerebellar tract in the cat. J. Physiol. (Lond.) 350: 599-614.

Triller, A., and H. Korn (1982) Transmission at a central inhibitory synapse. III. Ultrastructure of physiologically identified and stained terminals. J. Neurophysiol. 48: 708-736

Tuttle, R., S. Masuko, and Y. Nakajima (1986) Freeze-fracture study of the large myelinated club ending synapse on the goldfish Mauthner cell: Special reference to the quantitative analysis of gap junctions. J. Comp. Neurol. 246: 202-211.

Walmsley, B., E. Wieniawa-Narkiewicz, and M. J. Nicol (1985) The ultrastructural basis for synaptic transmission between primary muscle afferents and neurons in Clarke's column of the cat. J. Neurosci. 5: 2095-2106.

Wojtowicz, J. M., and J. L. Atwood (1985) Correlation of presynaptic and postsynaptic events during establishment of long-term facilitation at crayfish neuromuscular junction. J. Neurophysiol. 54: 220-230.

Yeh, J. Z., G. S. Oxford, C. H. Wu, and T. Narahashi (1976) Interactions of aminopyridines with potassium channels of squid axon membranes. Biophys. J. 16: 77-81.

Zucker, R. S. (1973) Changes in the statistics of transmitter release during facilitation. J. Physiol. (Lond.) 229: 787-810.

Zucker, R. S. (1974) Crayfish neuromuscular facilitation activated by constant presynaptic action potentials and depolarizing pulses. $\mathbf{J}$. Physiol. (Lond.) 241: 69-89.

Zucker, R. S., and L. O. Lara-Estrella (1979) Is synaptic facilitation caused by presynaptic spike broadening? Nature 278: 57-59.

Zucker, R.S., and N. Stockbridge (1983) Presynaptic calcium diffusion and the time courses of transmitter release and synaptic facilitation at the squid giant synapse. J. Neurosci. 3: 1263-1269. 